\title{
A head-mounted goggle-type video-oculography system for vestibular function testing
}

\author{
Youngsun Kong ${ }^{1}$, Suhwan Lee ${ }^{2}$, Jinseok Lee ${ }^{3}$ and Yunyoung Nam ${ }^{4 *}$ (D)
}

\begin{abstract}
Disorders of the vestibular system possibly decrease vision, causing abnormal nystagmus and dizziness. To diagnose abnormal nystagmus, various studies have been reported including the use of rotating chair tests and videonystagmography. However, these tests are unsuitable for home use due to their high costs. Thus, a low-cost video-oculography system is necessary to obtain clinical features at home. In this paper, we present a goggle-type low-cost video-oculography system using an infrared camera and Raspberry Pi board for tracking the pupils and evaluating a vestibular system. Horizontal eye movement is derived from video data obtained from an infrared camera and infrared light-emitting diodes, and the velocity of head rotation is obtained from a gyroscope sensor. Each pupil was extracted using a morphology operation and a contour detection method. Rotatory chair tests were conducted with our developed device. To evaluate our system, gain, symmetry, and phase were measured and compared System 2000.
\end{abstract}

Keywords: Infrared camera, Video-oculography, VOG, Videonystagmography, VNG

\section{Introduction}

During human locomotion, vision is disturbed by head perturbations [1]. The vestibulo-ocular reflex (VOR) helps the eyes fix on a target ahead, by rotating the moving eyes in the opposite direction [2]. This compensatory eye movement is induced by sensing angular acceleration in the semicircular canals of the vestibular system [3]. However, vestibular dysfunction in the form of blurring of vision in that a target slips from the fovea makes an eye movement pattern called nystagmus [4]. Visual acuity can decrease by $50 \%$ when an object is only $2^{\circ}$ from the center of the fovea, which can be caused by Nystagmus [5]. In addition, $35.4 \%$ of US adults aged 40 or older had some degree of vestibular dysfunction according to the survey from 2001 to 2004 [6]. There are two gold standard methods to diagnose nystagmus and evaluate the semicircular canals, rotatory chair tests are conducted on suspected patients [7]. These tests include

\footnotetext{
* Correspondence: ynam@sch.ac.kr

${ }^{4}$ Department of Computer Science and Engineering, Soonchunhyang University, Asan, South Korea

Full list of author information is available at the end of the article
}

the sinusoidal harmonic acceleration (SHA) test and the velocity step test. The SHA test measures factors that can infer a degree of dizziness by rotating a chair with frequencies of $0.01-0.64 \mathrm{~Hz}$ increased by two times in terms of sinusoidal velocity, and by measuring eye movement [7]. To analyze eye movement signals with the SHA test, three factors are calculated, which are gain, symmetry, and phase. Each feature represents overall responsiveness, the degree of symmetry between left and right stimuli, and time difference between head stimulus and eye movement. The velocity step test measures vestibular response decay by suddenly changing chair velocity and keeping it constant [7]. However, the SHA test is preferred generally due to limitations with the velocity step test that includes the need for a powerful chair, lower reliability, and more stress to the patient [7]. In this study, only SHA tests were conducted.

Electronystagmography (ENG) and scleral search coil systems (SSCSs) were used to collect eye movement signals [8, 9]. ENG measures movements of the eye by attaching electrodes around the nose, while ENG has the advantages of being able to make measurements while the eyes are closed; ENG has 


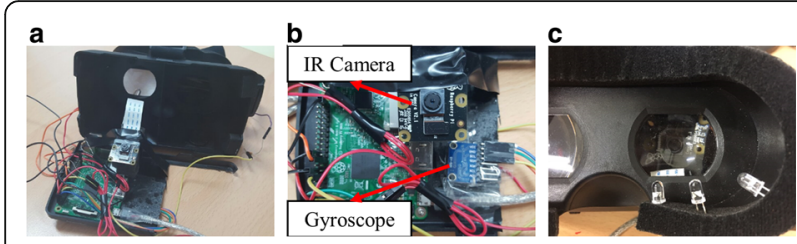

Fig. 1 Head-mounted goggles with an infrared camera. a Goggles inside. b Raspberry Pi 3. c IR LED

disadvantages of artifacts being caused by eye blinking, perspiration, or light, and difficulty in measuring vertical eye movement. Additionally, SSCS requires subjects to attach an annular contact lens to their eyes; although having a high accuracy and sampling rate, it can be worn for only $40 \mathrm{~min}$ and has a tendency to slip [10, 11]. With advances in computer vision technology, videonystagmography (VNG) has been used generally, although ENG data are still valuable [12]. VNG is a technology that can test whether dizziness is caused by inner ear disease; it uses an infrared camera to track the pupil in the dark. Advantages of VNG are its high accuracy and non-invasive nature, making its use common, although with high cost.

Various VNG researches using VNG have been conducted [13-17]. In [13], researchers suggested a new method to solve the problem of estimating the eye position in VNG analysis brought about by deformable contour methods. They suggested a method based on position, amplitude, and duration that could track saccade movement with high accuracy. In [14], a method of vestibular disease analysis for VNG applications was proposed, and new features were suggested based on Fisher's criteria for the diagnosis of nystagmus. In [15], researchers proposed a method for medical characteristic analysis with the displacement vectors of nystagmus using Gaussian mixture models (GMM). Video data were captured using

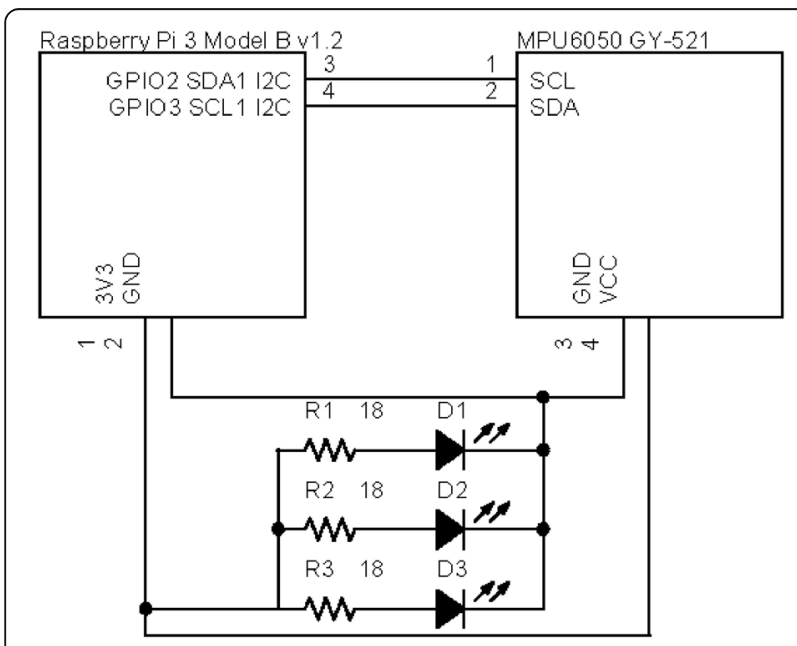

Fig. 2 PCB circuit of the goggles

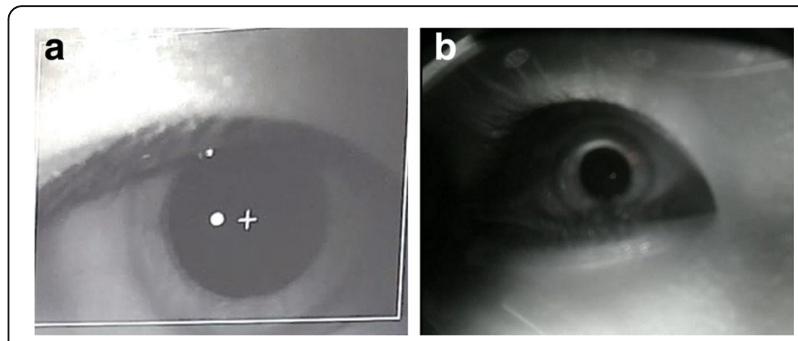

Fig. 3 Infrared images of a pupil. a System 2000. b Developed goggles

infrared cameras, and two-dimensional displacement vectors were calculated by comparing two adjacent frames. Then, GMM was applied to analyze the vectors. In [16], researchers compared the features extracted from the diameter and position of an eye recorded using VNG on patients and normal subjects. In [17], researchers analyzed VNG dataset based upon the fundamental measurements of normal and patients who have vestibular disorder by using a neural network to develop the assessment of vertigo symptom.

Various commercial VOG products have been developed $[18,19]$. They conduct and analyze results in SHA test, velocity step test, and optokinetic nystagmus test by measuring pupil movements with a rotatory chair and VOG to monitor vestibular loss, dizziness, and chronic imbalance. Also, they are highly accurate systems but with high cost and used only in hospitals.

In this paper, a pair of head-mounted goggles was built with an infrared camera and used with a rotatory chair to conduct SHA tests. Then, pupil coordinates were extracted from the video data. From the pupil data, gain, phase, and symmetry were calculated for measuring rapid eye movements. Finally, these factors were compared with System 2000 [18] results, which is a highly accurate system that measures pupil movements with a rotatory chair and infrared cameras and analyzes vestibular system, but it is expensive.
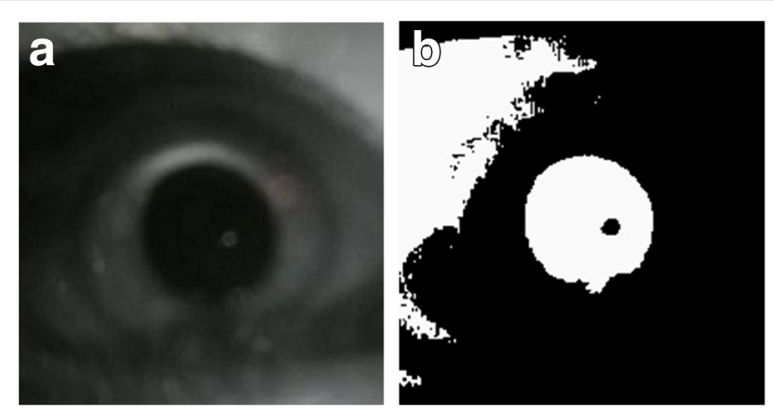

Fig. 4 Binarization. a Gray image. b Binary image 


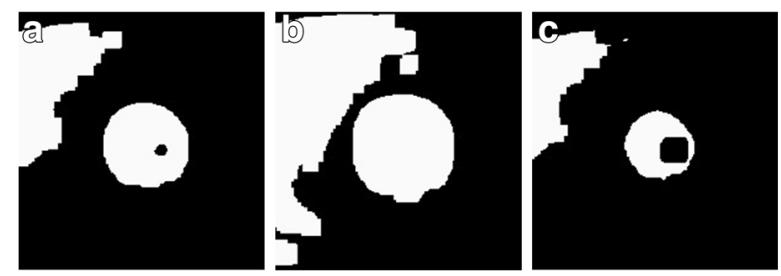

Fig. 5 Morphology operation. a Erode. b Dilation. c Erode and dilation

\section{Methods}

\subsection{Prototype}

Head-mounted goggles were designed for obtaining eye movement signals. Figure 1 shows a pair of headmounted goggles with an infrared camera and a gyroscope sensor. The goggles can record infrared videos of the right eye of the subject and measure rotatory velocity. To conduct a calibration, the participant can see the front with the left side of the goggles. The measured field of view is $32^{\circ}$. Three $850-\mathrm{nm}$ lightemitting diodes (LEDs) were attached to the goggles. To avoid noise caused by light reflexes, the LEDs were located at the bottom. The distances from the center were approximately $1.5,1.9$, and $2.9 \mathrm{~cm}$ from the left to the right LED. The prototype complies with International Standard IEC 60825-1 [20].

The prototype consists of an infrared camera, a gyroscope sensor, and infrared LEDs. 3D virtual reality (VR) glasses (Maruneuru, Korea) were used as the frame for the goggles. Figure 2 shows a printed circuit board (PCB) for the goggles. The Raspberry Pi 3 model B (Raspberry Pi Foundation, UK) [21] was used with the goggles for image processing and calculating features, due to its low cost, appropriate size, and fair image processing performance. Additionally, a Pi Camera (v2) without an IR filter, equipped with a IMX219 8-megapixel sensor (Sony, Japan) [22], was used to capture infrared images of

Table 1 Miss rate with morphology and no morphology

\begin{tabular}{lll}
\hline Subject & Miss rate with morphology & Miss rate without morphology \\
\hline 1 & $27.4 \%$ & $47.2 \%$ \\
2 & $41.6 \%$ & $92.5 \%$ \\
3 & $14.0 \%$ & $46.7 \%$ \\
4 & $7.1 \%$ & $78.9 \%$ \\
5 & $6.3 \%$ & $24.7 \%$ \\
6 & $30.5 \%$ & $75.6 \%$ \\
7 & $27.5 \%$ & $51.7 \%$ \\
8 & $18.3 \%$ & $66.0 \%$ \\
9 & $8.3 \%$ & $100.0 \%$ \\
10 & $8.3 \%$ & $82.6 \%$ \\
\hline
\end{tabular}

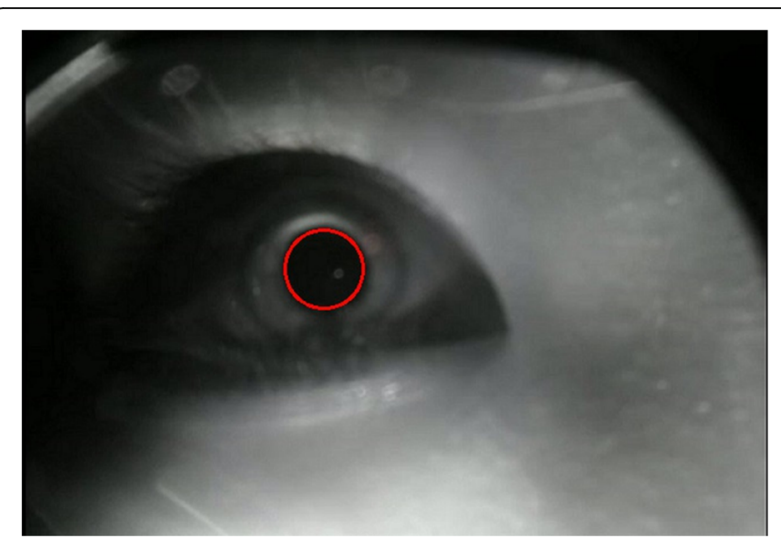

Fig. 6 Example of detected pupil

the eyes. A gyroscope MPU-6050 (InvenSense, USA) [23] was used to measure the angular velocity of the goggles.

\subsection{Pupil extraction}

To obtain horizontal eye movement, the pupil was extracted from video data obtained with the goggles and the System 2000; Fig. 3 shows screenshots of a healthy subject. Because pupils have a generally circular shape, the same method was used to both video data obtained from the goggles and the System 2000 with different threshold values of brightness and pupil size. The method includes noise removal and a circledetection algorithm.

To decrease the miss rate, the ROI was selected from raw images. The sizes of the ROIs for the developed goggles and the System 2000 were set at $200 \times 210$ and $400 \times 200$, respectively. Figure 4 shows a gray image and the result of image binarization. Because video data were obtained as RGB images, the gray channel was extracted from the infrared video data. The intensity of the pupil area is higher than that of the rest; thus, binary images were obtained from the extracted gray channel with manual threshold values for each subject. Binary images may contain noise caused by occlusion by the eyelids and eyelashes.

To solve the noise issue, a morphology operation was implemented (Fig. 5), whose kernel was set as 3 by 3 rectangular structuring element, and iterations

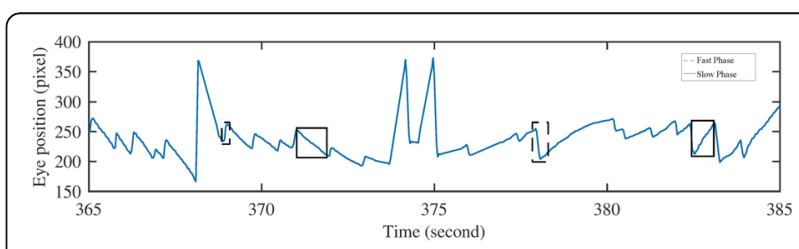

Fig. 7 Pupil movement signal (System 2000) 


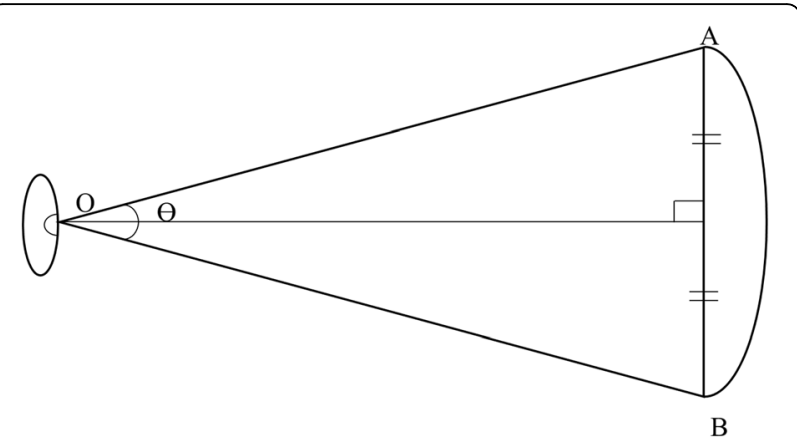

Fig. 8 Calibration. The distance between $\mathbf{a}$ and $\mathbf{b}$ is the actual diameter

were manually set for each subject. Table 1 shows the pupil miss rate, estimated from images where the morphology operation was and was not applied. The pupil miss rate is given by the following:

$$
\text { Miss rate }(\%)=\frac{\sum \text { False negative }}{\sum \text { Condition positive }} \text {, }
$$

Average of the miss rate is approximately $18.54 \%$.

Finally, FindContours function in OpenCV library was applied to detect a circular region. The contour candidates were selected by the following equations:

$$
\begin{aligned}
& \operatorname{abs}\left(1-\frac{w}{h}\right)<k, \\
& \operatorname{abs}\left(1-\frac{\mathrm{s}}{\pi \cdot\left(\frac{w}{2}\right)^{2}}\right)<k,
\end{aligned}
$$

where $w, h$, and $s$ represent width, height, and area of detected contours, respectively, and $k$ represents a constant value which was set to 0.6 or more. The minimum radius and area of the detected circle were set to 20 and 30 , respectively. A contour that has the minimum radius was chosen among the candidates. Figure 6 shows an example of an image with the method applied.

\subsection{Slow-phase eye movement}

Figure 7 shows a part of the eye movement signal obtained from the goggles. The dashed rectangles and the solid rectangles indicate fast-phase and slowphase eye movement, respectively. To extract features from eye-movement signals, the slow-phase velocity of the pupil should be calculated [24].

A degree per pixel value was assumed to determine the velocity of horizontal eye movement in degrees. Figure 8 shows a calibration method for obtaining degrees per pixel. The $\Theta$ value was calculated using a trigonometric function. Also, degrees per pixel for the goggles and the System 2000 were calculated by dividing $\Theta$ by $\overline{\mathrm{AB}}$. Finally, gain, symmetry, and phase were calculated from the signal.

\subsection{System 2000}

Slow-phase velocity is derived from the raw signal as shown in Fig. 9. First, the horizontal pupil movement signal was extracted from the infrared video data using a pupil extraction method. Missing frames caused by eye blinking were set as a negative number. In compensating for missing frames caused by eye blink artifacts, a linear spline algorithm was implemented. Moreover, a cubic spline algorithm

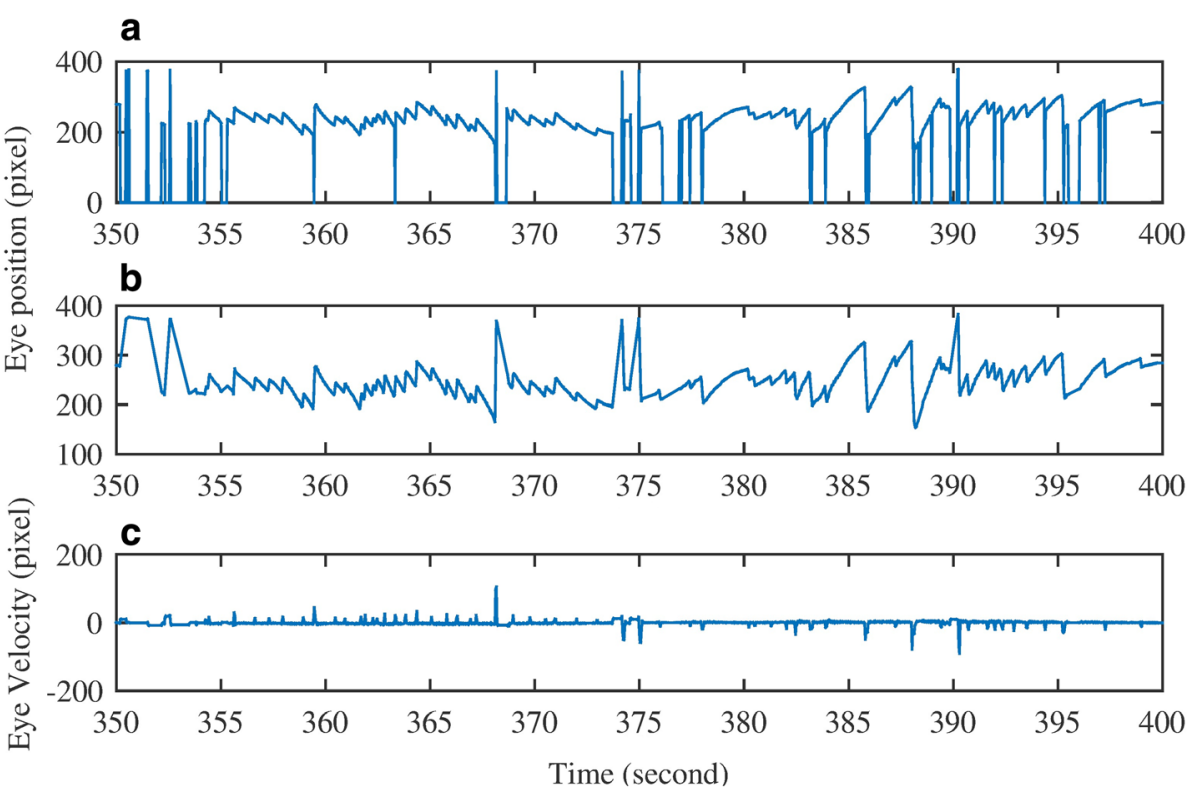

Fig. 9 Pupil movement signal (System 2000). a Raw signal. b Interpolated signal. c Derived pupil velocity 

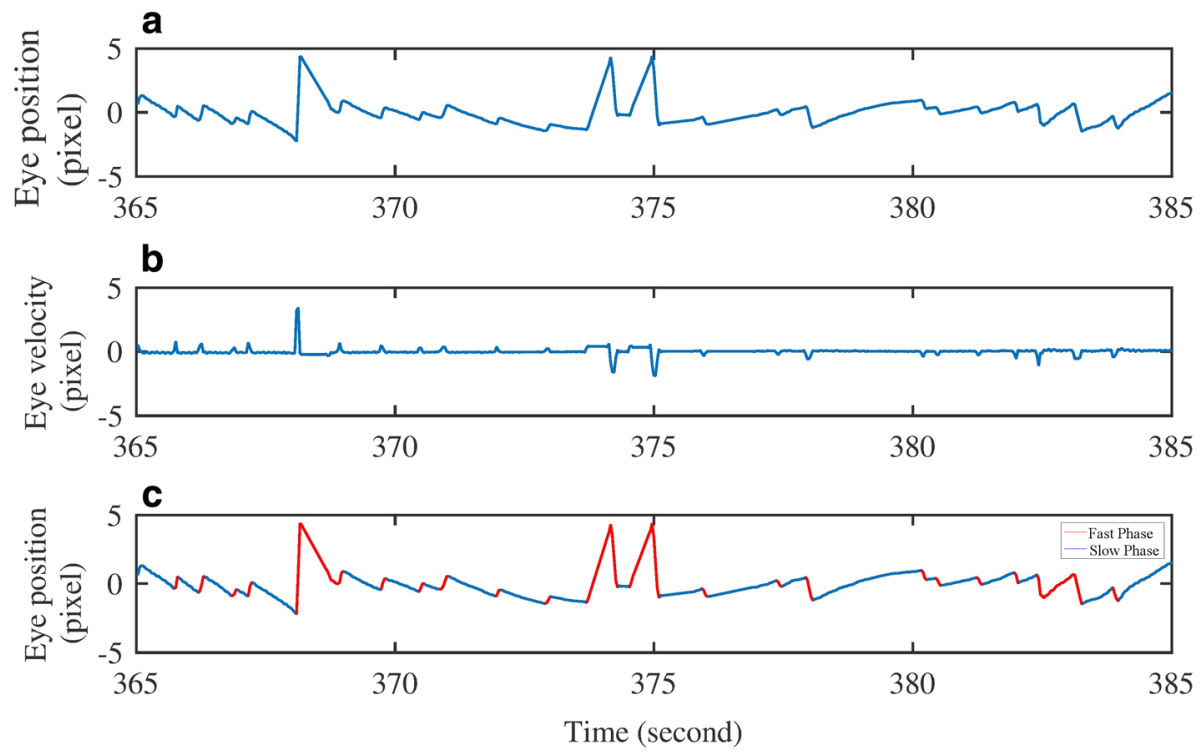

Fig. 10 Pupil movement signal (System 2000). a Normalized signal. b Derived signal. c Fast phase

was implemented to interpolate the signal to $30 \mathrm{~Hz}$ due to the irregular sampling rate (27-29 fps). Pupil velocity was also derived from the interpolated signal.

The fast phase of the signal was removed as follows. Since having a larger slope than the slow-phase velocity, the fast-phase velocity was determined based on slope degree. First, the obtained signal was normalized with a mean of 0 and a standard deviation of 1 . Then, the eye movement signal was derived and squared, to obtain the slope of the signal. Peaks were detected by a threshold value from the squared signal, which were manually set based on each velocity of chair. As shown in Fig. 10, the red line represents the fast phase obtained. Finally, the fast phase was removed from the derived signal.

After the fast-phase signal was eliminated, a linear spline algorithm was used. As shown in Fig. 11, low-pass filter was applied to remove noise from the velocity signals. The cut-off frequency of the low-pass filter was set as follows:

$$
f_{\text {low }}=k \cdot \frac{n}{\Delta t}
$$

where $k, n$, and $\Delta t$ represent a constant, the number of cycles, and the period, respectively, corresponding to the frequency of head movement multiplied by a constant. The constant values were manually set between 1 and 2 for each subject.

\subsection{Designed goggles}

The infrared camera used in the goggle design had a lower resolution and sampling rate than those of the System 2000. Thus, a different method was used to obtain the slow-phase signal and features calculated with the camera. Slow-phase velocity is derived from the raw signal as shown in Fig. 12. A negative number indicates that the pupil was not detected due to noise, such as eye blinking and low sampling rate. Then, raw signals were interpolated using a linear spline algorithm to remove missing frames. Because the sampling rate of the signals obtained from the goggles was irregular (25-30 fps), signals were interpolated using a cubic spline algorithm.

Figure 13 shows an example of a peak detection result from the pupil movement velocity signal. Since

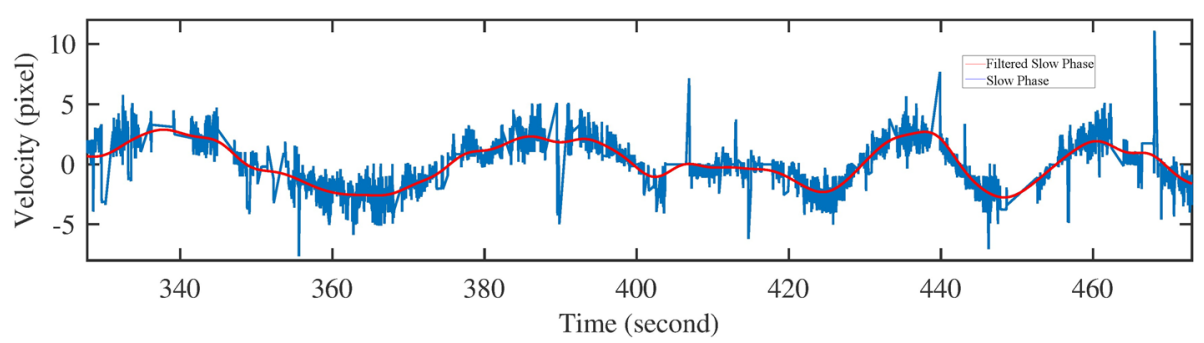

Fig. 11 Obtained slow phase and low-pass filter (System 2000) 


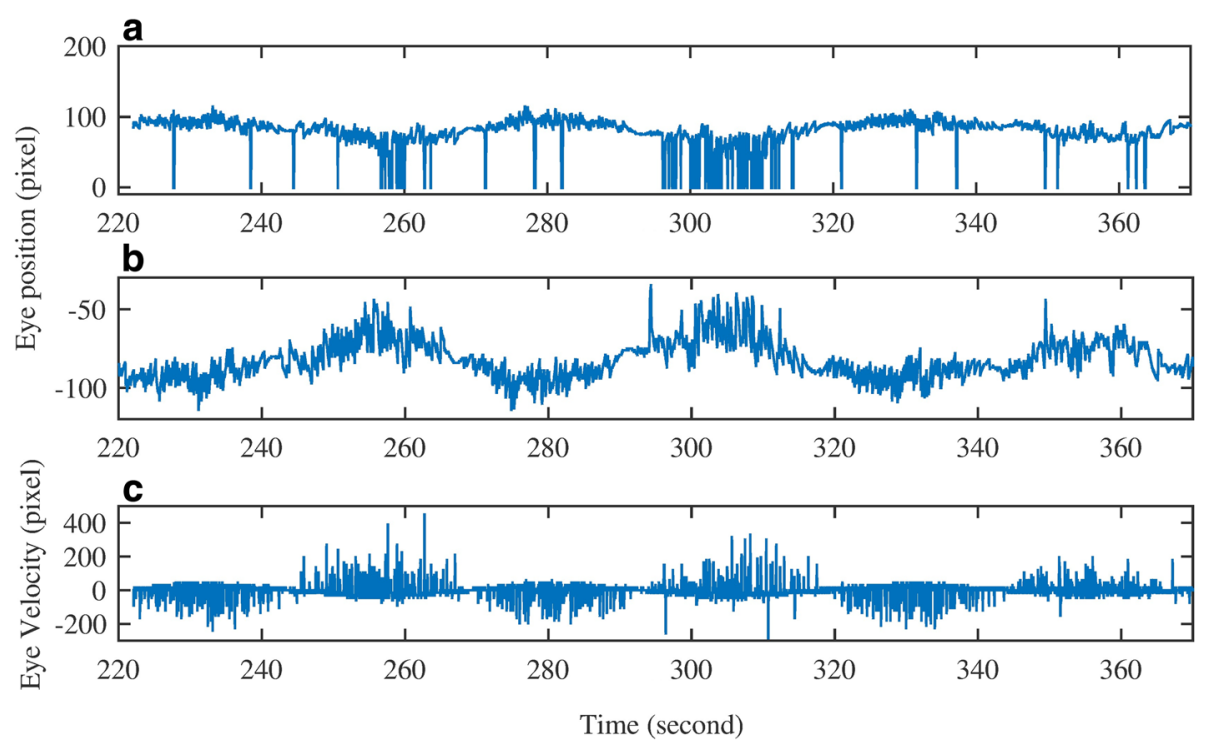

Fig. 12 Pupil movement signal (designed goggles). a Raw signal. b Interpolated signal. c Derived pupil velocity

the signals obtained between the designed goggles and System 2000 contain different levels of noise and have different frequencies, another method was used to find peaks. The 'Findpeaks' function in MATLAB was used to find each peak and the valley location. Yellow and red circles represent valley and high peaks of the signal, respectively. The velocity differences between adjacent peaks and valleys were calculated. Velocities that were less than the rotatory chair velocity were removed to eliminate fast-eye movement.

Moving-average and low-pass filters were then applied to remove noise. Figure 14 shows filtered signals with moving-average and low-pass filters. The same equation of cutoff frequency was applied, which is based on the frequency of head movement multiplied by a constant value.

\subsection{Gain, asymmetry, and phase}

Gain is the ratio of the amplitude of the eye movement to the amplitude of head stimulus [25]. Symmetry is indicated by comparable left and right responses when the same stimulus is applied [25].
Phase is the timing relationship between the initiation of head movement and the reflexive eye response [24]. Gain, symmetry, and phase are calculated as follows:

$$
\text { Gain }=\frac{\text { Amplitude of the maximum slow phase eye velocity }}{\text { Amplitude of the maximum stimulus velocity }},
$$

$$
\text { Symmetry }=\frac{b_{2}-b_{1}}{b_{2}+b_{1}} \times 100,
$$

$$
\text { Phase }\left(\phi^{\circ}\right)=360^{\circ} \cdot f \cdot \Delta t
$$

where $b_{1}$ and $b_{2}$ represent the maximum velocity of slow-phase eye movement in rotating to the left and right, respectively, $f$ represents the frequency of the eye movement signal, and $\Delta t$ represents the time difference between the maximum velocity of slow-phase eye movement and head movement.

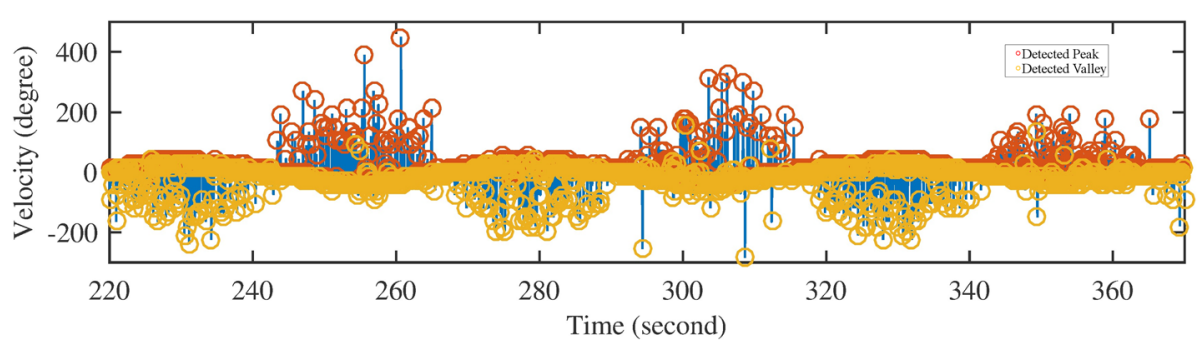

Fig. 13 Peak detection (designed goggles) 


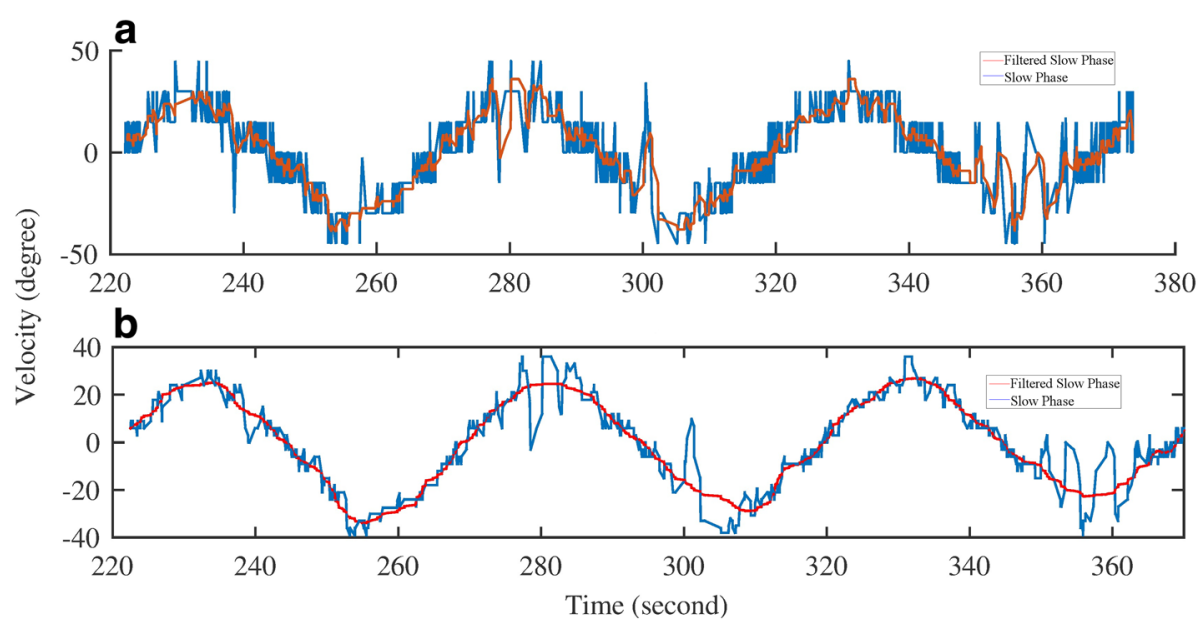

Fig. 14 Pupil movement signal (designed goggles). a Moving average. b Low-pass filter

\section{Results}

\subsection{Acquisition}

Data were collected from eight healthy non-smoking men and two women aged from 20 to 28 years. All subjects wore the developed goggles and System 2000 goggles, while seated on a rotatory chair (System 2000) in the sitting position (Fig. 15). Each experiment was performed seven times (from 0.01 to $0.64 \mathrm{~Hz}$ ). The chair was rotated with maximum velocity from $80^{\circ}$ to $20^{\circ}$ per second for 2 to 8 cycles (Table 2). The videos from the goggles were recorded at $600 \times 420$ resolution. The videos from the System 2000 were obtained using the built-in camera on a Galaxy S6 (Samsung, Korea) at $1920 \times 1080$ resolution by pointing the monitor of the computer, because no video data are ordinarily provided by the System 2000 and because of the limitation of accessing the computer. Both video data sets were recorded at $\sim 30 \mathrm{~Hz}$.

\subsection{System 2000}

Reference results were obtained from a System 2000 software itself. To evaluate the results, we calculated the error $\varepsilon$ for each frequency of gain, phase, and symmetry, as follows:

$$
\varepsilon=\frac{\operatorname{mean}\left(\operatorname{abs}\left(x-x_{\mathrm{est}}\right)\right)^{2}}{\operatorname{mean}(\operatorname{abs}(x))^{2}},
$$

where $x$ and $x_{\text {est }}$ represent the reference and estimated feature, respectively. Each error for gain, phase, and symmetry were measured among all subjects for each frequency. Figure 16 shows the median and interquartile range (IQR) of the errors measured from the results at each frequency. The mean errors for gain, symmetry, and phase were $0.81,17.35$, and 2.74 , respectively.
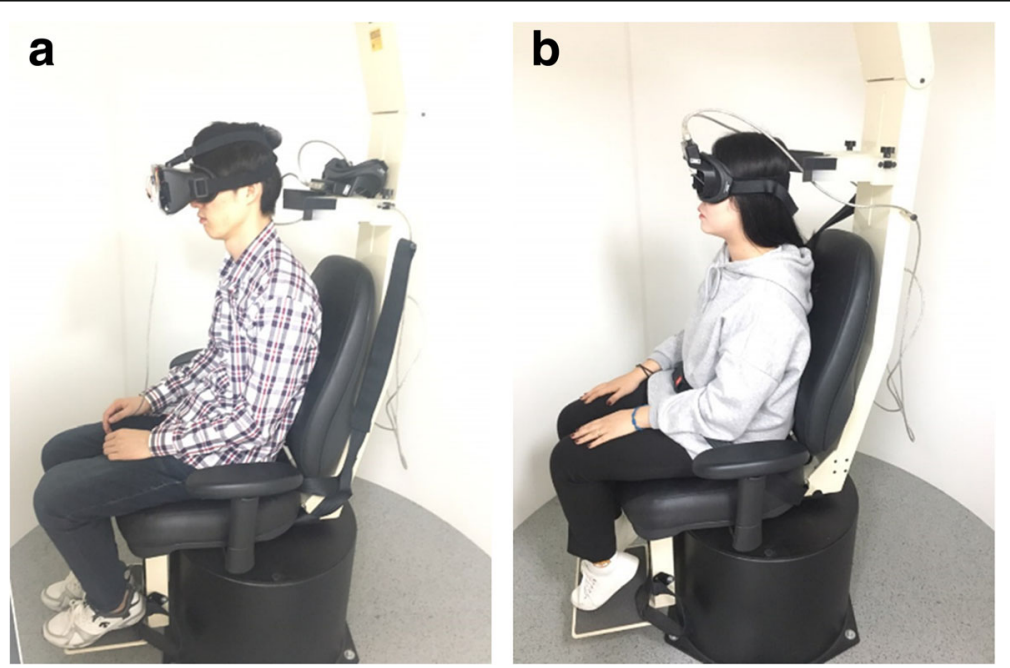

Fig. 15 Experimental setup. a Developed goggles. b System 2000 
Table 2 Experimental setup

\begin{tabular}{lll}
\hline Frequency of chair oscillation $(\mathrm{Hz})$ & Maximum velocity of chair (degree per second) & Period of chair oscillation \\
\hline 0.01 & 80 & 2 \\
0.02 & 70 & 3 \\
0.04 & 50 & 4 \\
0.08 & 40 & 5 \\
0.16 & 30 & 5 \\
0.32 & 20 & 6 \\
0.64 & & 8 \\
\hline
\end{tabular}

Figure 17 shows Bland-Altman and correlation plots of gain, symmetry, and phase with mean differences of $0.047,0.16$, and 0.029 , respectively; the solid lines and the dashed lines in part (b) of each figure represent the regression line and a Pearson correlation coefficient of 1 .

\subsection{Designed goggles}

It is hard to wear two goggles simultaneously without creating noise. Thus, we calculated the features and the coverage rate that measures how many results of the features are in the range of the reference data. The experimental setup was set to the same values used with the System 2000. The coverage rates for gain, symmetry, and phase were 66, 87, and $70 \%$, respectively.

\section{Conclusions}

This paper has presented a low-cost VNG using an infrared camera for the diagnosis of nystagmus by obtaining eye movement features. The device was developed using a pair of head-mounted goggles, a Raspberry Pi board, an infrared camera, and LEDs. To extract and track the pupil from video data obtained from the device, a morphology operation and a contour detection method were used. The miss rate was $18.54 \%$ when applying the morphology operation, whereas without applying it, the rate was $66.9 \%$. The horizontal eye movement signal was obtained, and its slow-phase velocity was calculated. Gain, symmetry, and phase were calculated from the velocity of horizontal eye movement and evaluated in 10 healthy subjects. Interpolation algorithms were used to detect missing signals caused by eye blinking in the subjects. Also, video data for the System 2000 are slightly tilted because of being captured by pointing the monitor of the computer and had minor oscillation noise caused by movements of subjects. Accordingly, the average IQR errors of gain and phase were 0.81 and 2.74, while that of symmetry was 17.35.

Because some images were misdetected due to noise caused by a low frame rate of IR camera and occlusion by eyelids and eyelashes, the relative high miss rate of pupil detection and the IQR errors was shown. Moreover, thresholds to detect pupil, to calculate degree per second, and frequency filtering factors are manually selected for each subject. Additionally, the number, location, and wavelength of the LEDs were not verified in the experiments. Also, relatively long missing frames with interpolation methods may cause minute distortion to signal and high IQR errors. Moreover, the developed goggles were not evaluated with a clinical device, because it is impossible to use two sets of goggles simultaneously without generating noise. In the future, more experiments will be conducted on more subjects including patients who have vestibular diseases for clinical tests. Also, we will evaluate the goggles

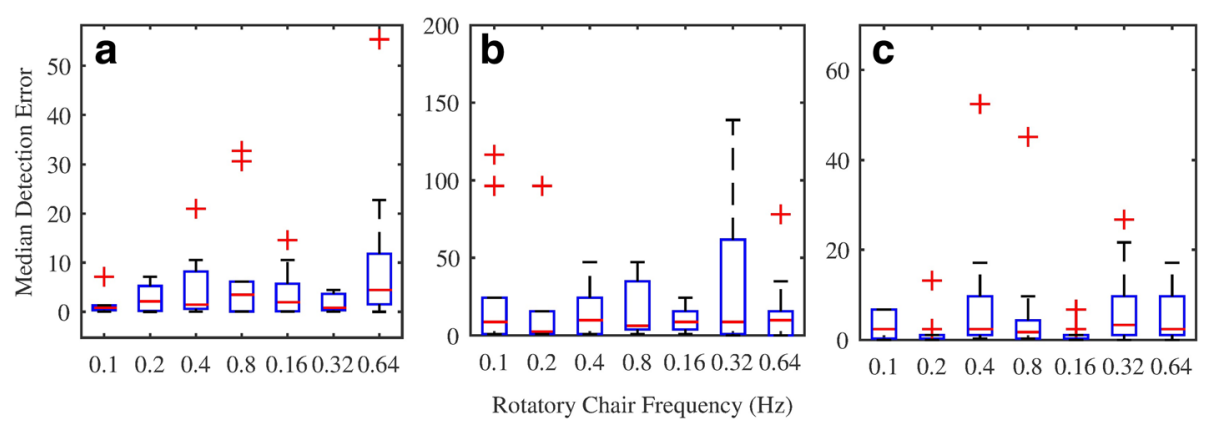

Fig. 16 Median and IQR errors measured from the gain, asymmetry, and phase at each frequency (all subjects). a Gain, b Asymmetry. c Phase 

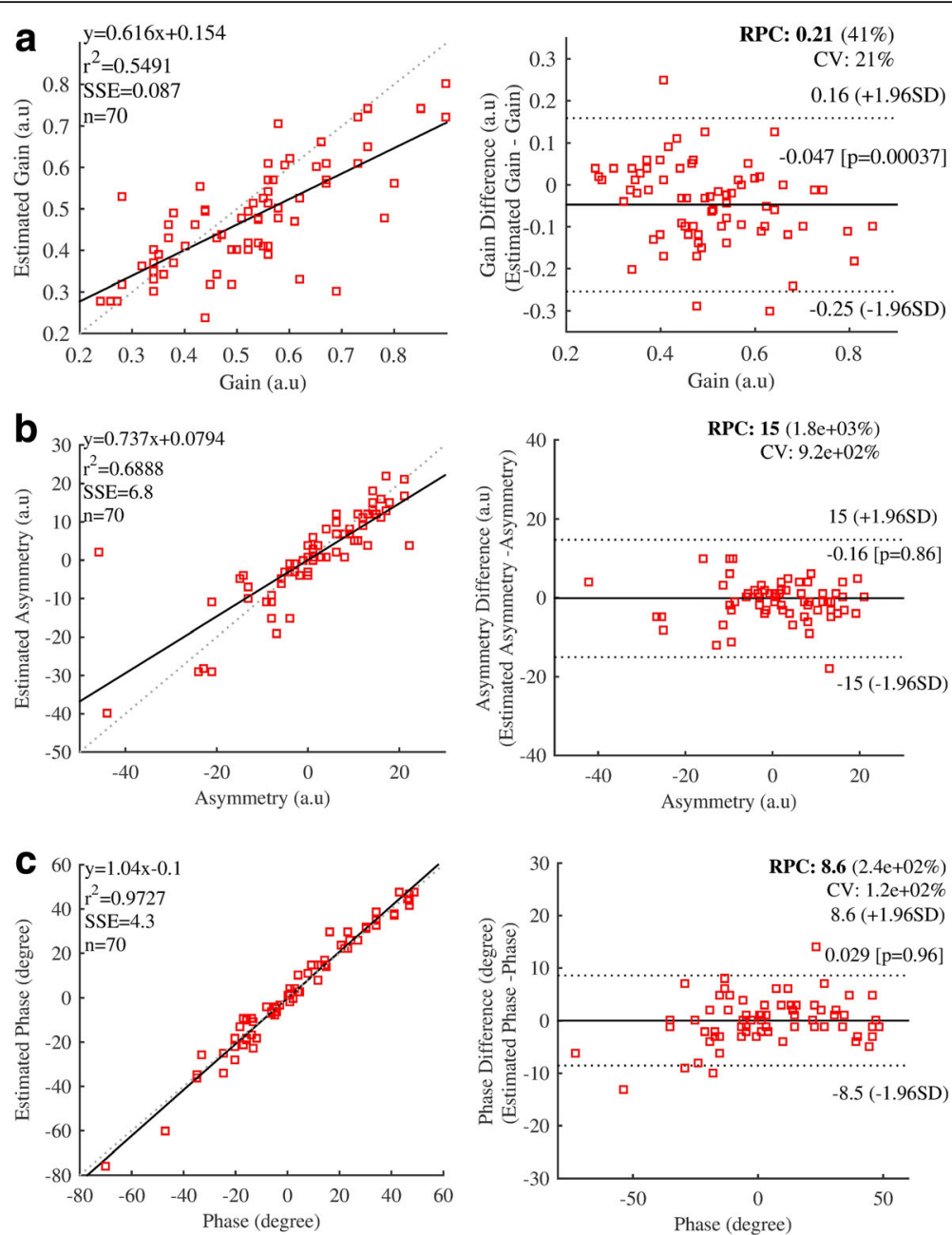

Fig. 17 Bland-Altman and correlation plots of gain, asymmetry, and phase. a Gain. b Asymmetry. c Phase

using other tests, such as the velocity step test to compare between the developed goggles and other clinical equipment. In addition, we will develop a VOG system that records pupil and angular velocity using an infrared camera and a gyroscope with any swivel chair at home.

\section{Abbreviations}

ENG: Electronystagmography; GMM: Gaussian mixture models; IQR: Interquartile range; LEDs: Light-emitting diodes; PCB: Printed circuit board; SHA: Sinusoidal harmonic acceleration; SSCSs: Scleral search coil systems; VNG: Videonystagmography; VOR: Vestibulo-ocular reflex; VR: Virtual reality

\section{Acknowledgements}

The authors would like to thank Namik Kim and Hyeonsu Lee for his valuable contribution to this project.

\section{Funding}

This work was supported by the Soonchunhyang University Research Fund and also supported by the "ICT Convergence Smart Rehabilitation Industrial Education Program" through the Ministry of Trade, Industry \& Energy (MOTIE), and Korea Institute for Advancement of Technology (KIAT).

\section{Availability of data and materials}

The datasets used and/or analysed during the current study are available from the corresponding author on reasonable request.

\section{Authors' contributions}

YK and YN conceived and designed the study. YK and SL performed the experiments. YK and YN wrote the paper. $J L$ and $Y N$ reviewed and edited the manuscript. All authors read and approved the final manuscript.

\section{Competing interests}

The authors declare that they have no competing interests.

\section{Publisher's Note}

Springer Nature remains neutral with regard to jurisdictional claims in published maps and institutional affiliations.

\section{Author details}

'Department of Biomedical Engineering, University of Connecticut, Storrs, CT, USA. ${ }^{2}$ Department of ICT Convergence Rehabilitation Engineering, Soonchunhyang University, Asan, South Korea. ${ }^{3}$ Department of Biomedical Engineering, Wonkwang University School of Medicine, Iksan, South Korea. ${ }^{4}$ Department of Computer Science and Engineering, Soonchunhyang University, Asan, South Korea. 
Received: 31 January 2018 Accepted: 11 April 2018

Published online: 27 April 2018

\section{References}

1. RJ Leigh, DS Zee, The Neurology of Eye Movements (Oxford University Press, USA, 2015)

2. RW Ditchburn, BL Ginsborg, Involuntary eye movements during fixation. J. Physiol. 119, 1 (1953)

3. JL Meiry, The Vestibular System and Human Dynamic Space Orientation (1966).

4. JM Epley, Positional vertigo related to semicircular canalithiasis. Otolaryngol Head Neck Surg. 112(1), 154-161 (1995)

5. DS Zee, The Neurology of Eye Movements [Electronic Resource] (Oxford University Press, Oxford, 1999)

6. Y Agrawal, JP Carey, CC Della Santina, MC Schubert, LB Minor, Disorders of balance and vestibular function in US adults: Data from the National Health and nutrition examination survey, 2001-2004. Arch. Intern. Med. 169, 938 (2009)

7. TC Hain, Rotatory Chair Testing (2016), https://www.dizziness-and-balance. com/testing/ENG/rchair.html. Accessed 15 Apr 2018

8. LB Jongkees, Electronystagmography. Hno 12, 325 (1964)

9. DA Robinson, A method of measuring eye movement using a scleral search coil in a magnetic field. IEEE Trans. Biomed. Electron. 10, 137 (1963)

10. ST Moore, T Haslwanter, IS Curthoys, ST Smith, A geometric basis for measurement of three-dimensional eye position using image processing. Vis. Res. 36, 445 (1996)

11. T Imai, K Sekine, K Hattori, N Takeda, I Koizuka, K Nakamae, K Miura, H Fujioka, T Kubo, Comparing the accuracy of video-oculography and the scleral search coil system in human eye movement analysis. Auris Nasus Larynx 32, 3 (2005)

12. MM Ganança, HH Caovilla, FF Ganança, Electronystagmography versus videonystagmography. Braz. J. Otorhinolaryngol. 76, 399 (2010)

13. AB Slama, AN Machraoui, M Sayadi, in Image Processing, Applications and Systems Conference (IPAS), 2014 First International. Pupil tracking using active contour model for videonystagmography applications (IEEE, Sfax, 2014), pp. 1-5

14. AB Slama, A Mouelhi, MA Cherni, S Manoubi, C Mbarek, H Trabelsi, M Sayadi, in Advanced Technologies for Signal and Image Processing (ATSIP), 2016 2nd International Conference on. Features extraction for medical characterization of nystagmus (IEEE, Monastir, 2016), pp. 292-296

15. Y Mao, Q Wang, J Miao, W He, in Biomedical Engineering and Informatics (BMEl), 2013 6th International Conference on. An application of Gaussian mixture models for medical characteristics analysis of nystagmus signals (IEEE, Hangzhou, 2013), pp. 18-23

16. H Salwa, W Turki, M Sayadi, in Image Processing, Applications and System Conferences (IPAS), 2014 1st International Conference, At Sfax. Study of the correlation between pupil position and diameter in video-nystagmotraphy for nystagmus analysis (2014), pp. 1-4

17. AB Slama, A Mouelhi, H Sahli, in Industrial Electronics Society (IECON), 2016 42nd Annual Conference on. A novel automatic diagnostic approach based on nystagmus feature selection and neural network classification (IEEE, Florence, 2016), pp. 5165-5170

18. Micromedical, System (2000), http://www.micromedical.com/Products/ Rotational-Chairs/. Accessed 15 Apr 2018

19. Vestlab, Vestlab OS. http://www.vestlab.de/produkt_en.html. Accessed 15 Apr 2018

20. International Electrotechnical Commission. Safety of Laser Products-Part 1: Equipment Classification and Requirements. Geneva, Switzerland. IEC-60825-1, (2014)

21. Raspberry PI Foundation, Raspberry Pi 3 model B. https://www.raspberrypi. org/products/raspberry-pi-3-model-b/. Accessed 15 Apr 2018

22. Sony, IMX219. http://www.sony-semicon.co.jp/products_en/new_pro/april_ 2014/imx219_e.html. Accessed 15 Apr 2018

23. InvenSense, MPU-6050. https://www.invensense.com/products/motiontracking/6-axis/mpu-6050/. Accessed 15 Apr 2018

24. MS Amin, Rotary Chair Testing: Overview, Indications, Equipment. (2016), https:// emedicine.medscape.com/article/1832765-overview. Accessed 24 Feb 2016.

25. PN Tandon, R Ramamurthi, Textbook of Neurosurgery, Third Edition, Three Volume Set (JP Medical Ltd, London, 2012)

\section{Submit your manuscript to a SpringerOpen ${ }^{\mathcal{O}}$ journal and benefit from:}

- Convenient online submission

- Rigorous peer review

- Open access: articles freely available online

- High visibility within the field

- Retaining the copyright to your article

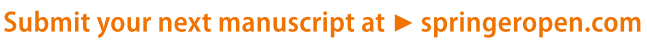

